\title{
Multiobjective Optimization of a Benfield HiPure Gas Sweetening Unit
}

\author{
Richard Ochieng, ${ }^{1,2}$ Abdallah S. Berrouk, ${ }^{1}$ and Ali Elkamel ${ }^{3}$ \\ ${ }^{1}$ Department of Chemical Engineering, Petroleum Institute, P.O. Box 2533, Abu Dhabi, UAE \\ ${ }^{2}$ Abu Dhabi Gas Industries Ltd., P.O. Box 66, Abu Dhabi, UAE \\ ${ }^{3}$ Department of Chemical Engineering, University of Waterloo, Waterloo, ON, Canada N2L 3G1
}

Correspondence should be addressed to Abdallah S. Berrouk; aberrouk@pi.ac.ae

Received 1 June 2013; Accepted 5 September 2013

Academic Editor: Jinwen Ma

Copyright (C) 2013 Richard Ochieng et al. This is an open access article distributed under the Creative Commons Attribution License, which permits unrestricted use, distribution, and reproduction in any medium, provided the original work is properly cited.

\begin{abstract}
We show how a multiobjective bare-bones particle swarm optimization can be used for a process parameter tuning and performance enhancement of a natural gas sweetening unit. This has been made through maximization of hydrocarbon recovery and minimization of the total energy of the process as the two objectives of the optimization. A trade-off exists between these two objectives as illustrated by the Pareto front. This algorithm has been applied to a sweetening unit that uses the Benfield HiPure process. Detailed models of the natural gas unit are developed in ProMax process simulator and integrated to the multi-objective optimization developed in visual basic environment (VBA). In this study, the solvent circulation rates, stripper pressure and reboiler duties are considered as the decision variables while hydrogen sulfide and carbon dioxide concentrations in the sweetened gas are considered as process constraints. The upper and lower bounds of the decision variables are obtained through a parametric sensitivity analysis of the models. The Pareto sets show a significant improvement in hydrocarbon recovery and a decent reduction in the heat consumption of the process.
\end{abstract}

\section{Introduction}

As global energy demand rises, natural gas now plays an important strategic role in world energy supply. It is the cleanest and most hydrogen rich of all the hydrocarbon energy sources and it has high energy efficiencies for power energy. Natural gas resources exploited and discovered are plentiful; however, they contain complex contaminants such as $\mathrm{CO}_{2}, \mathrm{H}_{2} \mathrm{~S}$, Mercaptans, and other sulfur compounds. Excessive amounts of these contaminants in natural gas streams will lead to low gas heating value and/or cause serious environmental hazards to the consumers. In LNG plants, large amounts of carbon dioxide and sulfur compounds may affect the quality of LNG products or pose serious operational problems in the cryogenic columns [1,2]. Therefore, one of the major purposes of the sweetening units is to purify raw natural gas to meet both sale gas and liquefaction specifications [3].

Unpredicted changes in reservoir conditions, combined with the tough market competition, have forced the natural gas sweetening business to adopt sophisticated optimization techniques to improve the purity of their products, increase production capacity, and minimize total energy requirement for units' operations.

Gas sweetening processes use complex facilities whose design and operation basically depend on many parameters including gas composition, flow rate, circulation rates, absorber temperatures and stripper pressures. Accurate modeling of such processes involving multicriteria decision making has inspired process engineers to acquire the best practices for process automation in the way to realize competitive returns on investment. Despite the rapid development in process automation, no systematic methodology can be employed to continuously guarantee optimality of process operation. However, routine update of performance can always be achieved.

Problems associated with the gas treating processes such as large energy consumption and limited gas recovery have led to the increasing focus on mathematical modeling and 
simulation of gas sweetening processes, often performed to understand effects of design and operation on process enhancement.

In most cases, optimization of such complex processes is achieved through parametric sensitivity analysis $[4,5]$. Such tedious procedures may be eliminated through the use of systematic approaches which guarantee global optimality of the process.

Previously, flow sheet optimization was achieved through calculation of inaccurate gradient derivatives of the process model generated inside modular process simulators [6]. The development of high speed computers has led to increasing focus on designing and optimizing chemical process using accurate and complex stochastic algorithms.

Further, the decreased computational time has also contributed to routinely solving real-world problems involving large, realistic nonlinear models often encountered in process engineering. Researchers such as Aroonwilas et al. [7] and Park and Kang [8] simulated $\mathrm{CO}_{2}$ removal with aqueous amine solutions such as DEA, MDEA, and DGA in a gas sweetening process. Rahimpour and Kashkooli $[9,10]$ developed a mathematical model for the absorption of $\mathrm{CO}_{2}$ by DEA-promoted potash solution and used it to examine the performance of a split flow absorber. More work on the modeling of the individual potassium carbonate and amine processes using computer simulations has been reported in the specialized literature [11-15].

Recently, engineering optimization has been heavily inclined towards nature-inspired and complex computational techniques such as evolutionary algorithms to achieve the necessary design and operation standards $[16,17]$. These complex and accurate optimization techniques have also been greatly motivated by the market availability of modern highperformance computers.

Optimization problems are often computationally expensive especially those involving multicriteria decision making processes. In practice, real-world engineering problems involve making decisions over multiple objectives such as maximizing production capacity, minimizing energy, maximizing reliability, or any other objectives which could be conflicting. For solving such problems accurately, researchers call for multiobjective optimization techniques which are nongradient based. The application of evolutionary optimization methods to solve complex engineering multi-objective optimization problems has been published in a number of articles [18-21].

In this paper, we show how a classical multi-objective bare-bone particle swarm optimization (MOBBPSO) can be effectively utilized in process parameter tuning and performance analysis for a natural gas sweetening unit through hydrocarbon recovery maximization and energy minimization. The two objectives are combined and solved as a single objective problem through the weighted sum method described by Marler and Arora [22].

Once optimization basis is determined, it is necessary to determine process variables to be optimized. Process variables are the variables that affect the values of the objective functions. These can be divided into decision and dependent variables. Decision variables are determined while dependent variables are influenced by process constraints. In this study, concentrations of $\mathrm{H}_{2} \mathrm{~S}$ and $\mathrm{CO}_{2}$ in the purified gas were considered as the dependent variables. A parametric sensitivity study is carried out on the process model to determine the upper and lower bounds of the decision variables. The bounds are necessary to enable the optimizer to create a search space which obeys the necessary rules of thumb.

The optimization code developed in Visual Basic for Application (VBA) environment and then integrated with ProMax process simulator to tune the solvent circulation rates, reboiler duties, and regeneration pressures of the gas sweetening units, so that energy consumption and hydrocarbon recovery are optimized.

This paper is divided into six sections. Section 2 introduces multi-objective optimization through the weighted sum approach and also describes a brief theory on barebones particle swarm optimization. Section 3 describes the Benfield HiPure process and the use of ProMax to develop its model. This section also contains a comparison of simulation results with plant data obtained from ADGAS. Section 4 describes the parametric sensitivity analysis which explains how the constraints in Section 5 are obtained. The optimization results are presented and discussed in Section 6 . Conclusions on this study including possible future work are discussed in Section 7 of this article.

\section{Multiobjective Optimization}

A general multi-objective problem can be expressed as a set of equations below:

$$
\text { Minimize } f(\bar{x})=\left[f_{1}(\bar{x}), f_{2}(\bar{x}), \ldots, f_{k}(\bar{x})\right]
$$

Subject to

Constraints:

$$
\begin{aligned}
& g_{i}(\bar{x}) \leq 0, \quad i=1,2, \ldots, m \\
& h_{j}(\bar{x})=0, \quad j=1,2, \ldots, q
\end{aligned}
$$

Bounds:

$$
a_{l} \leq x_{l} \leq b_{l}, \quad l=1,2, \ldots, p,
$$

where $\bar{x}=\left[x_{1}, x_{2}, \ldots, x_{p}\right]^{T}$ is the vector of decision variables, $f_{i}: \mathfrak{R}^{n} \rightarrow \mathfrak{R}, i=1,2, \ldots, k$, are the objective functions, $g_{i}, h_{j}: \Re^{n} \rightarrow \mathfrak{R}, i=1,2, \ldots, m, j=1,2, \ldots, q$, are the constraint functions of the problem and $a_{l}, b_{l}: \Re^{n} \rightarrow \Re, l=$ $1,2, \ldots, p$, are the lower and upper bounds of the decision variables, respectively.

Such multiple objective problems are often appearing in many process engineering problems [23]. In most cases, these objectives are generally conflicting and hence preventing simultaneous optimization of the individual objectives.

The most common approach to multi-objective optimization is the weighted sum method (5) which transforms the multiple objective problem into a single objective engineering problem $[22,23]$. Objectives are allocated weights and added 
to form a single objective problem (maximization objectives take a negative sign). Consider

$$
f(\bar{x})=\sum_{i=1}^{k} w_{i} f_{i}(\bar{x}) .
$$

Multi-objective optimization in process plants has been recently reported by Wu et al. [21], Bernier et al. [24], Li et al. [25], Montazer-Rahmati and Binaee [26], Sun and Lou [27], and Shadiya et al. [28]. Genetic algorithm [19] has always been the most common global optimization algorithm employed in optimizing process plants. Recently particle swarm optimization has gained more interest in obtaining global optimum of nonconvex problems [29]. Poli et al. [30] discussed an overview on the development and application of particle swarm optimization to real-world problems, and Reyes-Sierra and Coella [31] presented a survey on use of multi-objective particle swarm optimizers in solving multiple objectives problems. Here, one of the improved versions of particle swarm optimization (PSO) commonly known as bare-bone particle swarm optimization (BBPSO) will be utilized to optimize the natural gas process. PSO has undergone several changes since its introduction in 1995 [32]. One of these changes was the use of the Gaussian normal distribution of mean and standard deviation to update particle position. In BBPSO, the exploration search is facilitated by the standard deviation which tends to zero as the search space progresses. More information on the development and application of BBPSO is discussed in the work published by Zhang et al. [33].

\section{Case Study}

3.1. Benfield HiPure Process of Natural Gas Sweetening. The Benfield HiPure process was first described in 1974 by Benson and Parish [34]. This process consists of the Benfield process and an amine unit downstream to scrub the remaining traces of acid gases. The integrated schematic of the Benfield HiPure process used in ADGAS plant at Das Island is shown in Figure 1.

The hot potassium carbonate absorption system comprises a split flow absorber and a regenerator with no side draws. The carbonate absorber and regenerator are both vertical packed bed columns. The treated gas from the carbonate absorber is fed directly into the amine absorber. The DEA amine system comprises absorber and stripper columns which are vertical and made of a stack-structured packing. The rich solution from the absorber is pumped to the DEA regenerator that has no condenser. The overhead gas (from DEA regenerator) is fed to the middle of the carbonate regenerator which does have a condenser. Liquid from the carbonate regenerator condenser is fed to the top of the DEA regenerator as reflux.

Sweet gas exiting the DEA absorber will undergo further processing before it is sent for LNG processing. The stripped acid gases from both the carbonate and DEA regenerators proceed to a sulfur recovery unit (SRU), where they are processed to produce molten liquid sulfur. Table 1 gives the typical operating data of the ADGAS plant.
TABLE 1: Typical ADGAS operating data.

\begin{tabular}{lc}
\hline Parameter & Value \\
\hline Feed Gas Flow Rate (MMSCFD) & 476.9 \\
Feed Gas Temperature $\left({ }^{\circ} \mathrm{C}\right)$ & 25.0 \\
Feed Gas Pressure (barg) & 52.1 \\
$\mathrm{H}_{2} \mathrm{~S}$ Feed Gas Composition (\%) & 4.7 \\
$\mathrm{CO}_{2}$ Feed Gas Composition (\%) & 2.1
\end{tabular}

Hot Potassium Carbonate Unit

Circulation Rate $\left(\mathrm{m}^{3} / \mathrm{hr}\right)$

Main: 343.5

Split: 1292.2

Lean Solvent Temperature $\left({ }^{\circ} \mathrm{C}\right)$

Main: 81.8

Lean Solvent Pressure (barg)

Split: 117.0

$\mathrm{K}_{2} \mathrm{CO}_{3}$ Concentration ( $\mathrm{wt} \%$ )

51.4

30.0

Promoter Concentration (DEA) (wt\%)

3.0

Amine Unit

Circulation Rate $\left(\mathrm{m}^{3} / \mathrm{hr}\right)$

109.8

Lean Solvent Temperature $\left({ }^{\circ} \mathrm{C}\right)$

49.9

Lean Solvent Pressure (barg)

53.7

DEA Concentration (wt\%)

20.0

3.2. Simulation Results. The process calculations were completed using ProMax V3.2 [35]. The electrolytic property package was used to predict the $\mathrm{H}_{2} \mathrm{~S}$ and $\mathrm{CO}_{2}$ absorption in both potassium carbonate and amine units of the Benfield HiPure process. The TSWEET kinetics model in ProMax was used to predict the $\mathrm{CO}_{2}$-amine/carbonate kinetic reactions taking place in all absorbers. TSWEET kinetics was developed by Bryan Research and Engineering for the purpose of accurately calculating the relatively slow absorption of $\mathrm{CO}_{2}$ by amine solutions.

Table 2 gives a comparison of the simulation results and the operating data. As can be seen, there is a close match between the simulation results with plant data. In the next section, a sensitivity analysis study will be described in order to show the effect of various operating conditions on the performance of the process and to motivate the optimization study that will be discussed in a later section of this paper.

\section{Sensitivity Analysis}

Sensitivity analysis gives the best approach to investigate the effect of process parameters on performance of a chemical plant. Here, a scenario tool was developed in ProMax, and the effect of change in decision variables is monitored with respect to the required gas specification (process constraint) while compromising the respective rules of thumb.

Figure 2 shows the effect of change in the carbonate circulation rate with composition of $\mathrm{H}_{2} \mathrm{~S}$ and $\mathrm{CO}_{2}$ in the purified gas. As shown in the figure, decreasing the carbonate circulation rate beyond $1400 \mathrm{~m}^{3} / \mathrm{hr}$ will rapidly cause an increase in $\mathrm{H}_{2} \mathrm{~S}$ and $\mathrm{CO}_{2}$ composition in the purified gas, hence violating the constraints of not more than 1 ppmv $\mathrm{H}_{2} \mathrm{~S}$ and 50 ppmv $\mathrm{CO}_{2}$. 


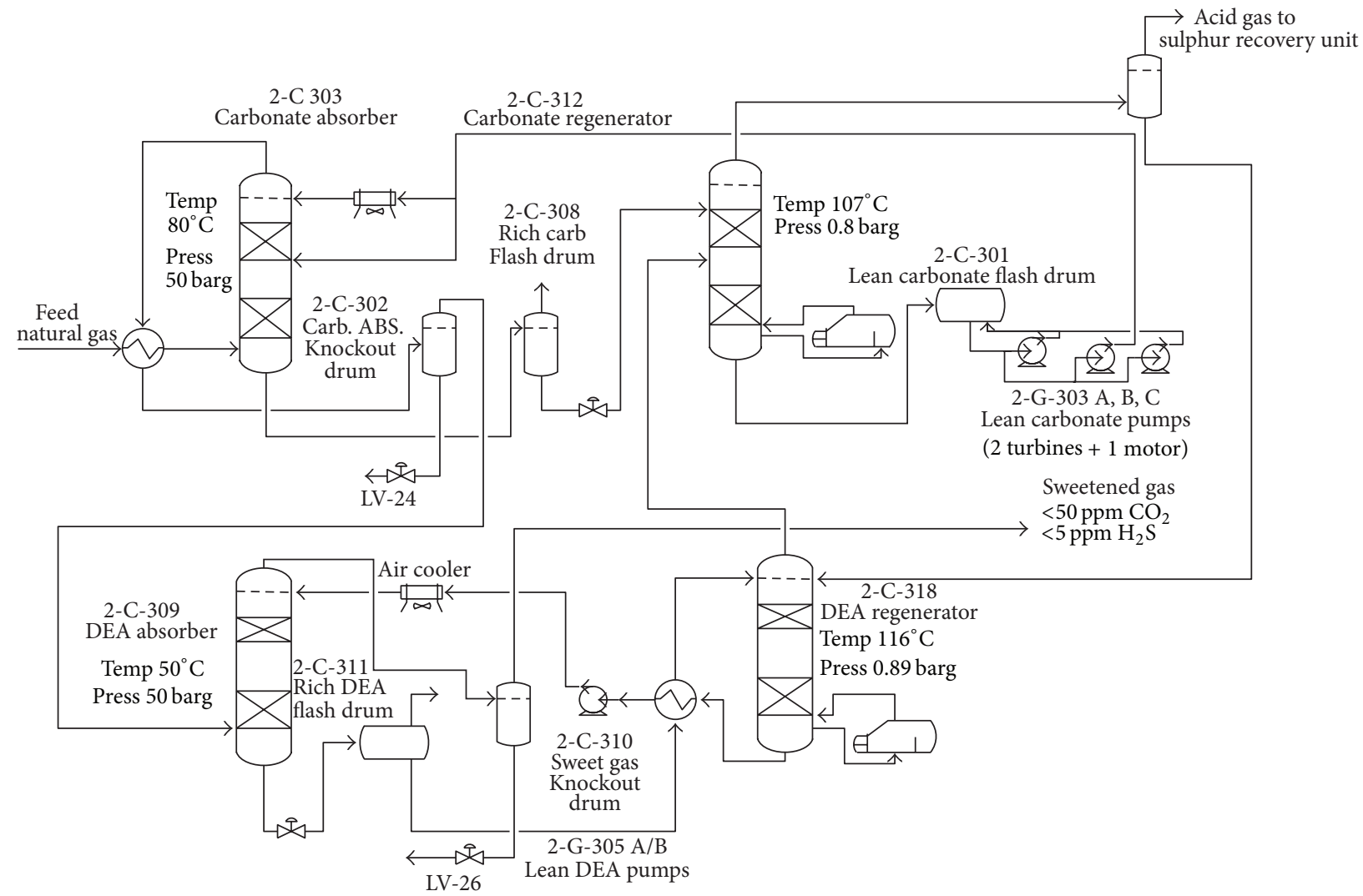

FIgURE 1: ADGAS’ Train number 3 gas treating plant: Schematic of the Benfield HiPure process.

TABLE 2: Comparison of hot carbonate section with plant data.

\begin{tabular}{|c|c|c|c|c|c|}
\hline \multirow{2}{*}{ Components } & \multirow{2}{*}{ Sour gas feed } & \multicolumn{2}{|c|}{ Carbonate absorber Overhead product } & \multicolumn{2}{|c|}{ Amine absorber Overhead product } \\
\hline & & Plant data & Simulation & Plant data & Simulation \\
\hline $\mathrm{CO}_{2}$ (ppmv) & 47000.0 & 574.1 & 570.0 & 19.00 & 25.00 \\
\hline $\mathrm{H}_{2} \mathrm{~S}$ (ppmv) & 21000.0 & 707.4 & 683.0 & 0.40 & 0.40 \\
\hline Nitrogen (v/v\%) & 2.1 & 2.1 & 2.3 & 2.30 & 2.31 \\
\hline Methane (v/v\%) & 81.4 & 85.4 & 86.2 & 85.90 & 87.11 \\
\hline Ethane (v/v\%) & 5.6 & 6.9 & 5.9 & 6.90 & 6.00 \\
\hline Propane (v/v\%) & 2.7 & 3.1 & 2.9 & 3.10 & 2.90 \\
\hline i-Butane (v/v\%) & 0.4 & 0.6 & 0.4 & 0.60 & 0.39 \\
\hline n-Butane (v/v\%) & 0.6 & 0.4 & 0.6 & 1.00 & 0.64 \\
\hline i-Pentane (v/v\%) & 0.1 & 0.2 & 0.1 & 0.20 & 0.20 \\
\hline n-Pentane (v/v\%) & 0.3 & 0.3 & 0.3 & 0.30 & 0.30 \\
\hline
\end{tabular}

On the other hand, a carbonate circulation rate of $1700 \mathrm{~m}^{3} / \mathrm{hr}$ or more will produce sweet gas with almost the same gas specification. Therefore, 1400 and $1700 \mathrm{~m}^{3} / \mathrm{hr}$ will be considered as the lower and upper bounds of the carbonate circulation rate, respectively. Figure 3 shows that decreasing the carbonate section reboiler energy beyond $40 \mathrm{Gcal} / \mathrm{hr}$ will instantly cause the product gas to go off spec; therefore, this will act as the lower bound for this decision variable. To minimize waste of energy, the upper bound may be constrained at $50 \mathrm{Gcal} / \mathrm{hr}$ since at this value, all the sweet gas produced meets the required specification.
In a similar way, Figure 4 is considered to choose the upper and lower bounds on the circulation rate of diethanolamine (decision variable $x_{3}$ ) as 90 and $110 \mathrm{~m}^{3} / \mathrm{hr}$, respectively. From Figure 5, the lower and upper bounds on the amine section reboiler duty may lead 10 and $12 \mathrm{Gcal} / \mathrm{hr}$, respectively.

Figures 6 and 7 show the variation of column stripping pressures with $\mathrm{H}_{2} \mathrm{~S}$ and $\mathrm{CO}_{2}$ composition in the sweet gas. Figure 6 shows that operating the carbonate stripper at pressures above 0.8 barg may lead to producing sweet gas which violates constraints, and to enable a moderate stripping 


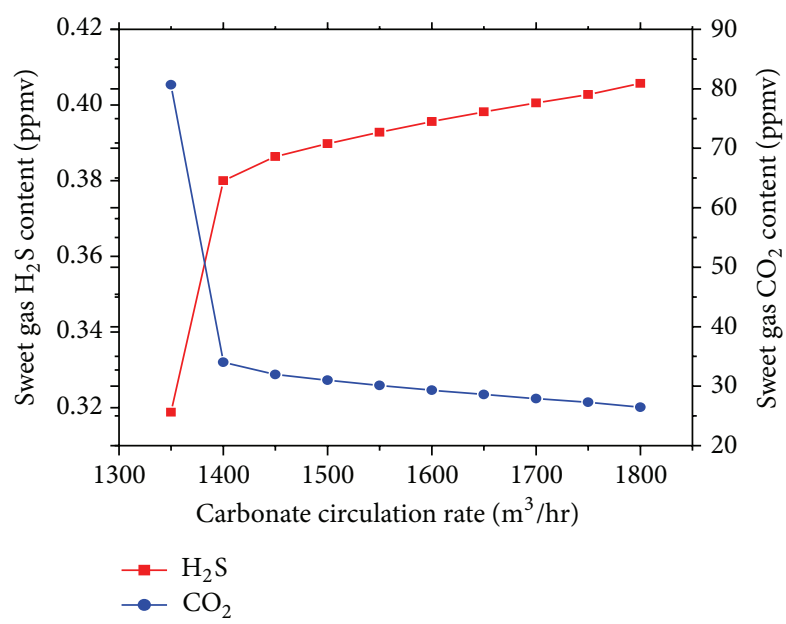

Figure 2: Effect of $\mathrm{K}_{2} \mathrm{CO}_{3} /$ DEA circulation rate on $\mathrm{CO}_{2}$ and $\mathrm{H}_{2} \mathrm{~S}$ composition in sweet gas.

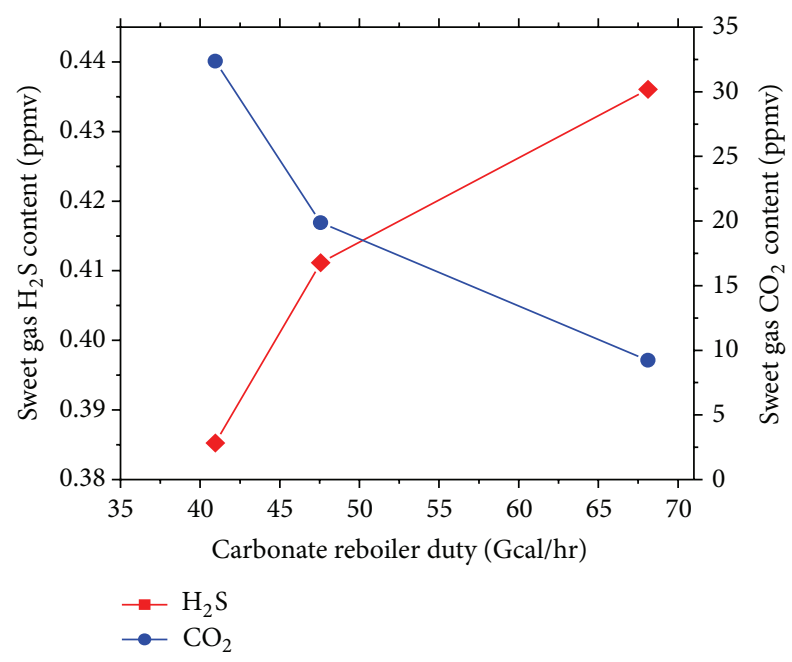

FIGURE 3: Effect of the reboiler duty of the carbonate section on $\mathrm{CO}_{2}$ and $\mathrm{H}_{2} \mathrm{~S}$ composition in sweet gas.

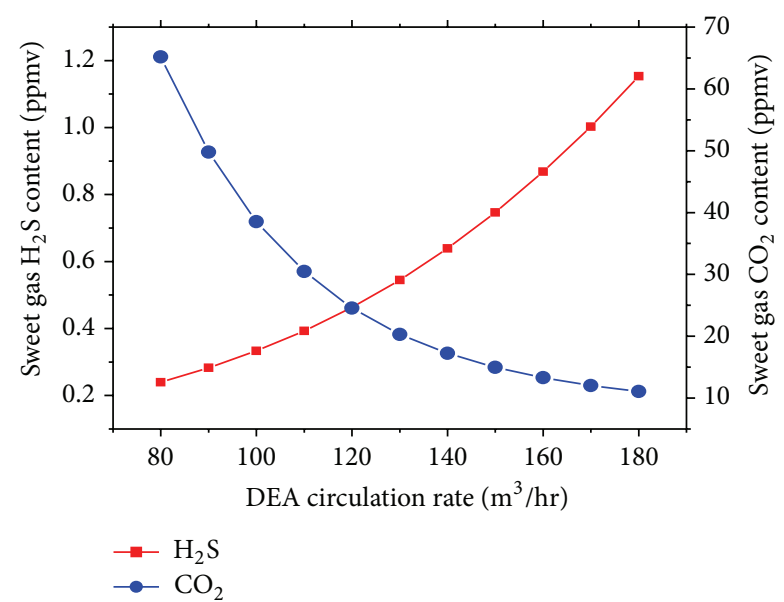

FIgURE 4: Effect of DEA circulation rate on $\mathrm{CO}_{2}$ and $\mathrm{H}_{2} \mathrm{~S}$ composition in sweet gas.

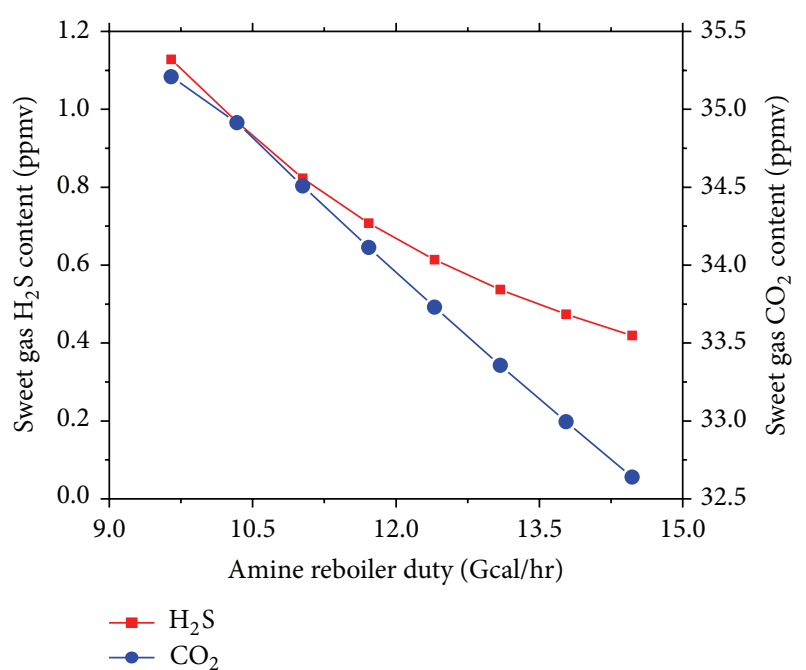

FIGURE 5: Effect of the reboiler duty of the amine section on the $\mathrm{CO}_{2}$ and $\mathrm{H}_{2} \mathrm{~S}$ composition in sweet gas.

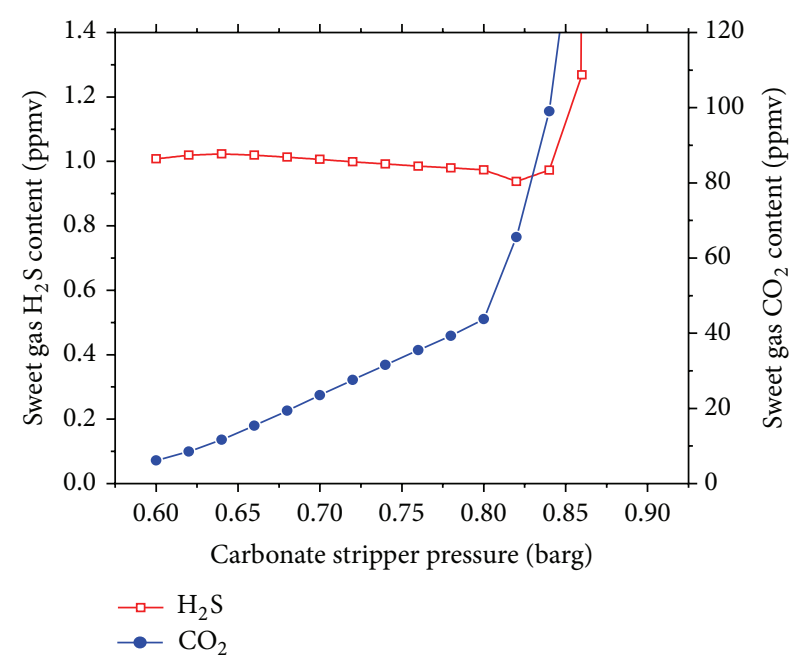

FIGURE 6: Effect of carbonate stripper pressure on $\mathrm{CO}_{2}$ and $\mathrm{H}_{2} \mathrm{~S}$ composition in sweet gas.

efficiency, 0.7 barg is considered as the lower limit decision variable.

Figure 7 shows that the variation of the amine stripper pressure with the composition of hydrogen sulfide shows a reduction in $\mathrm{H}_{2} \mathrm{~S}$ removal and exhibits a maximum increase at about $1 \mathrm{barg}$, and increasing the stripper pressure beyond this region rapidly increases the $\mathrm{H}_{2} \mathrm{~S}$ removal. On the other hand, an increase in stripper pressure continuously decreases the amount of $\mathrm{CO}_{2}$ in the sweet. Therefore, 0.9 barg and 1.2 barg are considered as the lower and upper limit on the decision variable, respectively. The lower limit pressure on the amine stripper is always expected to be greater than the upper limit for the carbonate stripper pressure since the overhead acid gas from the amine regenerator is directly feeding the carbonate stripper. 


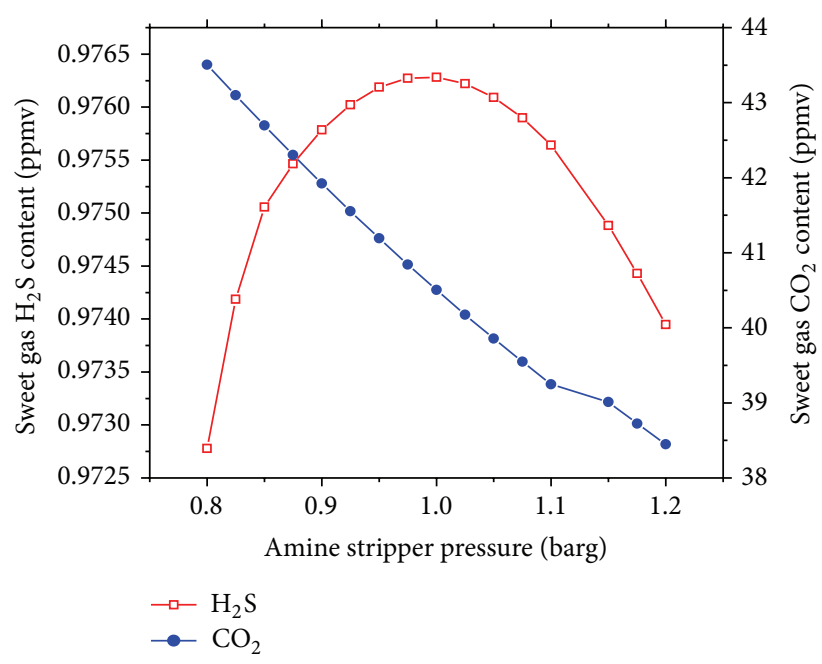

Figure 7: Effect of amine stripper pressure on $\mathrm{CO}_{2}$ and $\mathrm{H}_{2} \mathrm{~S}$ composition in sweet gas.

\section{Process Optimization}

The optimization of the gas sweetening unit involves two objectives: maximization of hydrocarbon recovery and minimization of the heat required to run the plant perunit time. This problem considers no variation in sour gas conditions (flow rate, temperature, pressure, and composition).

The mathematical expression of the objective functions of the optimization problem can be cast as

Maximize $f_{1}(\bar{x})=$ Hydrocarbon Recovery,

$$
\text { Hydrocarbon Recovery }=\left(\frac{M_{S}-M_{a}}{M_{f}}\right) * 100 \% \text {, }
$$

Minimize $f_{2}(\bar{x})=$ Total Heat,

Total Heat

$$
=\sum\left(Q_{\text {Reboilers }}+Q_{\text {Pumps }}-Q_{\text {Coolers }}-Q_{\text {Condensers }}\right) .
$$

The first objective function represented by (6) is the percentage amount of sweet gas produced from the gas facility excluding $\mathrm{CO}_{2}$ and $\mathrm{H}_{2} \mathrm{~S}$. This is also used as the measure of hydrocarbon content $\left(\mathrm{C}_{1}\right.$ to normal- $\left.\mathrm{C}_{5}\right)$ since the feed gas contained no traces of mercaptans or another sulfur compounds. $M_{S}$ and $M_{a}$ are the mass flow rate of the sweetened gas and acid gas $\left(\mathrm{CO}_{2}\right.$ and $\left.\mathrm{H}_{2} \mathrm{~S}\right)$, respectively. $M_{f}$ is the mass flow rate of the sour gas fed to the gas facility. Equation (7) represents the second objective function which is the total heat required to produce $M_{S} \mathrm{~kg}$ of sweet gas in a given unit of time.

Gas sweetening plants require high amounts of energy supply to produce ultrasweet gas needed in LNG plants for safe process operation and high efficient hydrocarbon separation in the downstream operations.

To maximize the sweet gas purity (high hydrocarbon content), higher amounts of heat need to be applied to absorb a substantial amount of the acid gases. However,
TABLE 3: The upper and lower bound of the operating variables.

\begin{tabular}{lcc}
\hline Process parameter & $\mathrm{LB}_{i}$ & $\mathrm{UB}_{i}$ \\
\hline$x_{1}$ & 1400 & 1700 \\
$x_{2}$ & 40 & 50 \\
$x_{3}$ & 90 & 110 \\
$x_{4}$ & 10 & 12 \\
$x_{5}$ & 0.7 & 0.8 \\
$x_{6}$ & 0.9 & 1.2 \\
\hline
\end{tabular}

due to the high costs of energy to run the plant facility, energy minimization is often recommended to reduce the plant operating costs. Therefore, due to the two conflicting objectives, process operators often strive for the search of a trade-off between energy minimization and hydrocarbon (pure sweet gas) maximization.

Here, these two objectives are modeled and combined through the weighted sum method to form a single objective. The weights are selected interactively to produce preference information needed during the progressive optimization process.

The constraints of the optimization problem are

$$
\begin{gathered}
y_{\mathrm{H}_{2} \mathrm{~S}}<1, \\
y_{\mathrm{CO}_{2}}<50, \\
L B_{i} \leq x_{i} \leq U B_{i} .
\end{gathered}
$$

Equations (8) and (9) provide constraints on the maximum composition of $\mathrm{H}_{2} \mathrm{~S}$ and $\mathrm{CO}_{2}$ allowable in the sweetened gas, respectively. Ultrasweet gas of utmost 1 and 50 ppmv of $\mathrm{H}_{2} \mathrm{~S}$ and $\mathrm{CO}_{2}$, respectively, is used as feed to the liquefied natural gas plant. $L B_{i}$ and $U B_{i}$ in (10) are the upper and lower bounds on the various conditions of the process.

These bounds are imposed on the $\mathrm{K}_{2} \mathrm{CO}_{3} / \mathrm{DEA}$ Circulation rate $\left(x_{1}\right)$, carbonate section reboiler duty $\left(x_{2}\right)$, DEA circulation rate $\left(x_{3}\right)$, amine section reboiler duty $\left(x_{4}\right)$, carbonate stripper pressure $\left(x_{5}\right)$, and amine stripper pressure $\left(x_{6}\right)$. From the simulation results and the sensitivity analysis of the previous section, appropriate bounds that define the search space are summarized in Table 3 . These bounds are chosen based on a physical understanding of the problem so as not to compromise desired operating conditions.

5.1. Simulation Optimization Framework. Simulation optimization entails finding optimal settings of the input variable(s), that is, values of $x_{1}, x_{2}, \ldots, x_{6}$, which optimize the output variable(s) of the simulation model. Optimizers designed for simulation embody the principle of executing separately from the model. In such a context, the optimization problem is defined outside the complex system. The output of the simulation model is used by an optimizer to provide new input values of the decision variables as the search for the optimal solution proceeds. On the basis of both current and past evaluations, the method decides upon a new set of input values.

Provided that a feasible solution exists, the optimization procedure ideally carries out a special search until an 


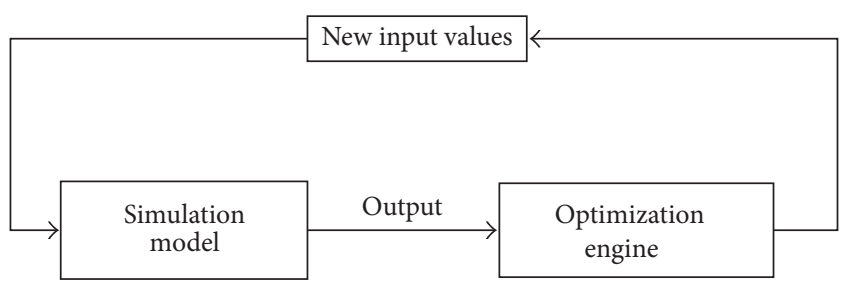

FIGURE 8: Integrated simulation optimization framework.

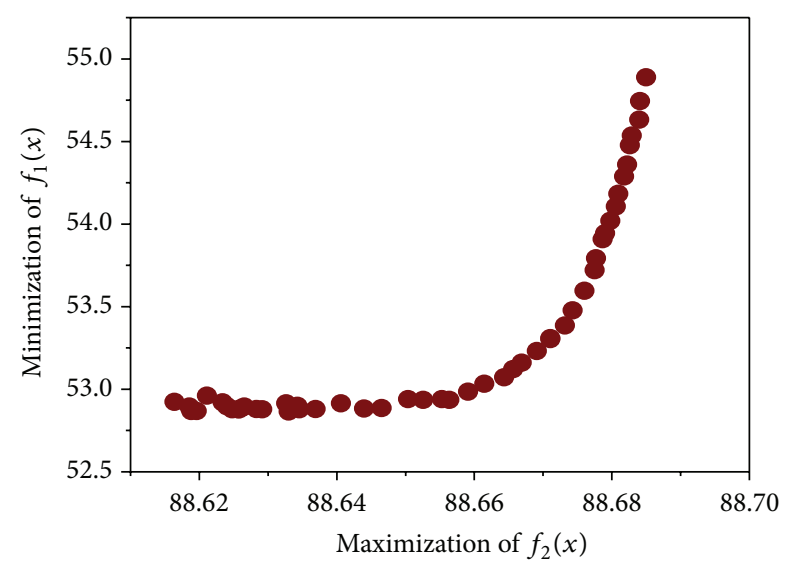

Figure 9: Pareto optimal front obtained from the simultaneous optimization of $f_{1}(\bar{x})$ and $f_{2}(\bar{x})$.

appropriate termination criterion is satisfied. A sequentiallike approach can be employed by coupling the solution of the optimization problem to an optimization routine (Figure 8). Every time the optimization routine needs a function evaluation by this approach, a call is made to the simulation model. In this work, optimization was performed through the barebone particle swarm optimization as discussed next.

5.2. Particle Swarm Optimization. PSO is inspired by the social behavior of some biological organisms, especially the group's ability of some animal species to locate a desirable position at the given area. It was proposed first by Kennedy and Eberhart [32]. In the PSO, a swarm consists of a set of particles, and each particle represents a potential solution of an optimization problem. Particles are placed in the search space of the function, and each evaluates the objective function at its current location.

Each individual in the basic particle swarm is composed of $d$, the dimensions of the optimization problem (number of decision variables), the current position $x_{i}$, the previous best position $p_{i}$, and the velocity update $v_{i}$. Kennedy [36] conducted some experiments using PSO variants, which drop the velocity term and update a particle using Gaussian normal distribution of mean and standard deviation. The use of Gaussian normal distribution in the basic PSO algorithm to update the particle is known as bare-bones particle swarm optimization (BBPSO). Unlike the PSO, the BBPSO is parameter-free and is suitable for those real application problems where the information on parameters such as inertia weights and acceleration coefficients of particles is lacking or hard to obtain.

The bare-bones particle swarm optimization (BBPSO) proposed by Kennedy [36] is a simple version of PSO. The BBPSO algorithm does not use the particle velocity but uses a Gaussian sampling based on Pgbest ${ }^{k}$ and Pbest $_{i}^{k}$, and the position equation is replaced by

$$
x_{i}^{d, k+1}=N\left(\frac{\left(\text { Pbest }_{i}^{d, k}+\text { Pgbest }^{d, k}\right)}{2}, \mid \text { Pbest }_{i}^{d, k}-\text { Pgbest }^{d, k} \mid\right) \text {. }
$$

Equation (11) shows that the position of each particle is randomly selected from the Gaussian distribution with the average of the personal best position and the global best position.

In BBPSO, the exploration search is facilitated by $\sigma_{i}^{d, k}$ term, which tends to zero as the search progresses, and more emphasis will be put on the exploitation of the search. Kennedy [36] further proposed an alternative version of BBPSO also, referred to as BBExp in the present paper, where the $d$ th dimension of the $i$ th particle is updated as follows:

$$
\begin{aligned}
& x_{i}^{d, k+1} \\
& =\left\{\begin{array}{cc}
N\left(\frac{\left(\text { best }_{i}^{d, k}+\text { Pgbest }^{d, k}\right)}{2}, \mid \text { best }_{i}^{d, k}-P \text { best }^{d, k} \mid\right) \\
\text { if } U[0,1]<0.5 \\
\text { best }_{i}^{d, k} & \text { Otherwise. }
\end{array}\right.
\end{aligned}
$$

Equation (12) justifies that since there is $50 \%$ chance that the $d$ th dimension of the particle changes to the corresponding pbest position, the BBExp inclines to search for pbest positions. Thus, BBExp is said to be biased towards exploiting the pbest positions as shown in Algorithm 1.

\section{Results and Discussion}

Figure 9 shows a Pareto set of optimal solutions obtained from the formulated problem $((6)-(12))$. The figure shows that increasing heat of the process $\left(f_{2}\right)$ will reduce the amount of $\mathrm{H}_{2} \mathrm{~S}$ and $\mathrm{CO}_{2}$ in the sweet gas $\left(f_{1}\right)$, meaning that the gas will contain more of the hydrocarbons than the acid gases.

Each point on the Pareto set is associated with a set of decision variables. A unique solution can be obtained using high-level decision making by management. The closeness of the Pareto set shows that a unique solution can also be easily generated using this method.

The percentage recovery of hydrocarbons may be interpreted as a small change along the hydrocarbon recovery axis. However, if translated into an equivalent amount of gas per unit time, the change is significant. That is to say, a $0.1 \%$ increase in hydrocarbon recovery is equivalent to approximately 9.4 tonnes of hydrocarbon per day.

Figures 10 and 11 show a comparison of the Pareto optimal solutions with the nonoptimal operating region. The 


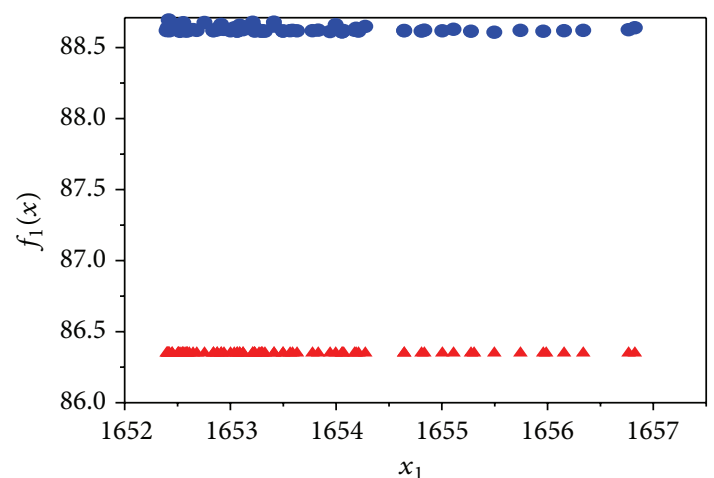

(a)

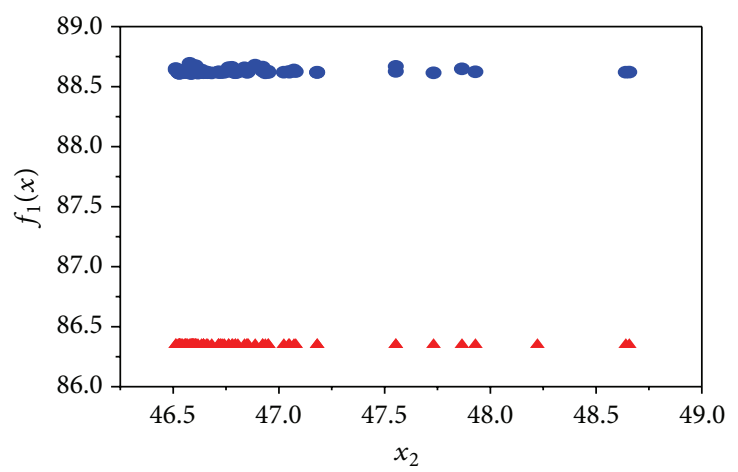

(c)

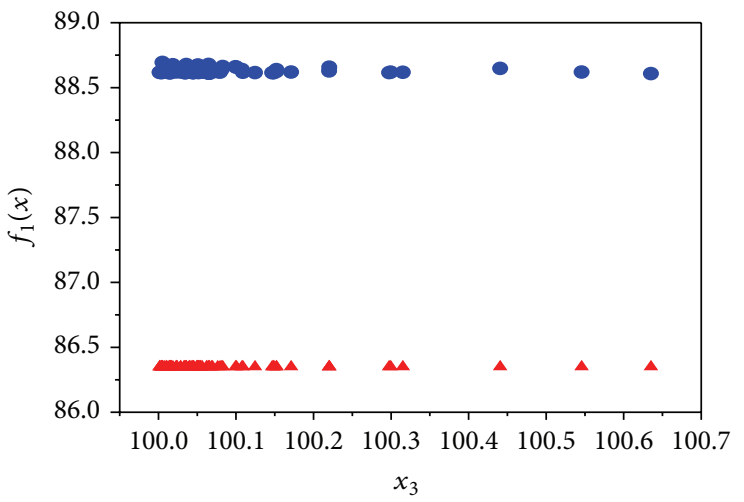

(e)

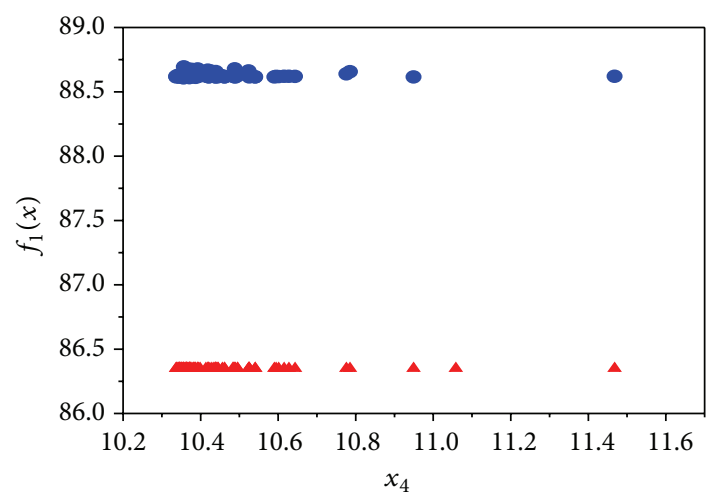

- Optimal

- Non-optimal

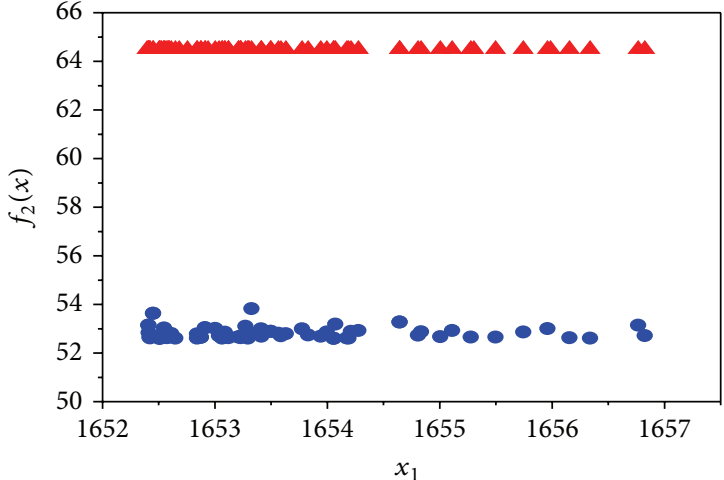

(b)

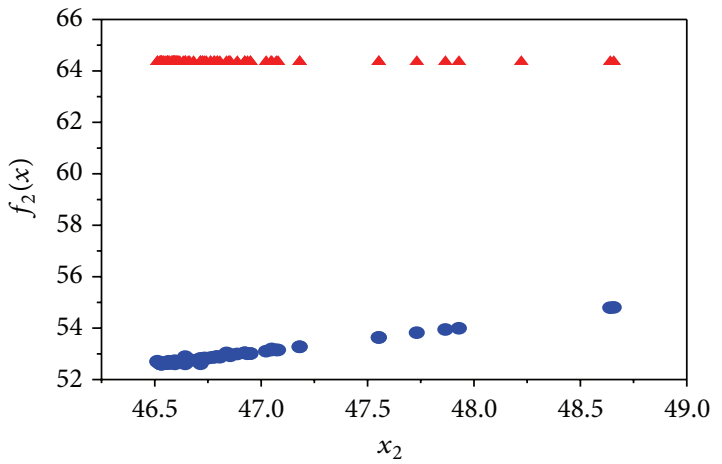

(d)

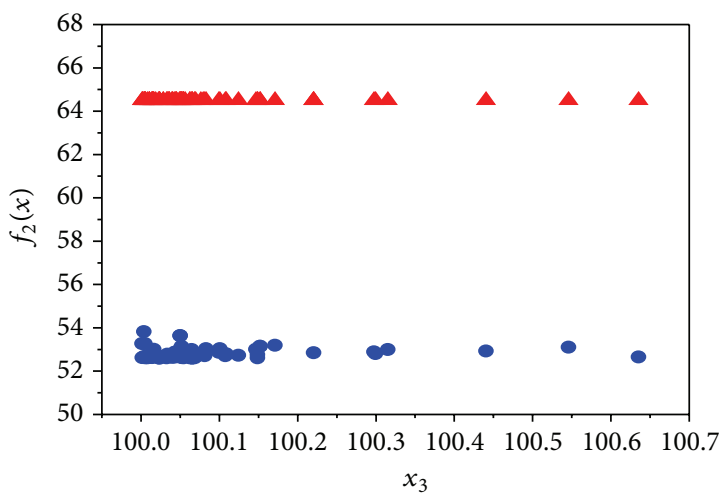

(f)

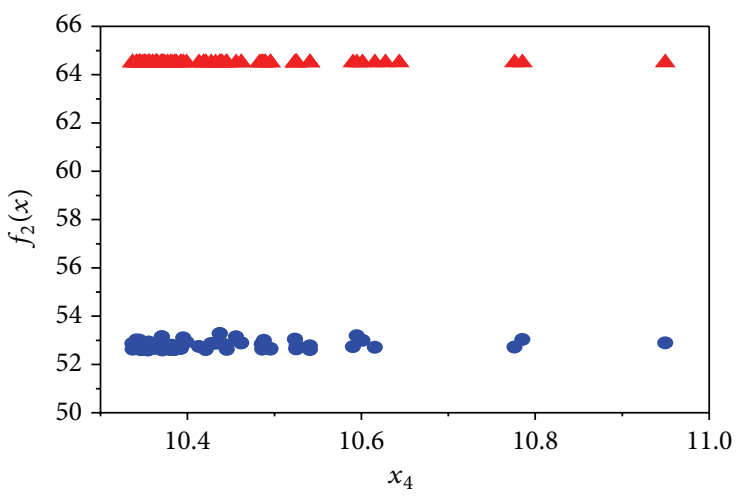

- Optimal

A Non-optimal

(g)

(h)

Figure 10: Pareto optimal set with regard to decision variables $x_{1}, x_{2}, x_{3}$, and $x_{4}$ with objectives $f_{1}(x)$ and $f_{2}(x)$. 


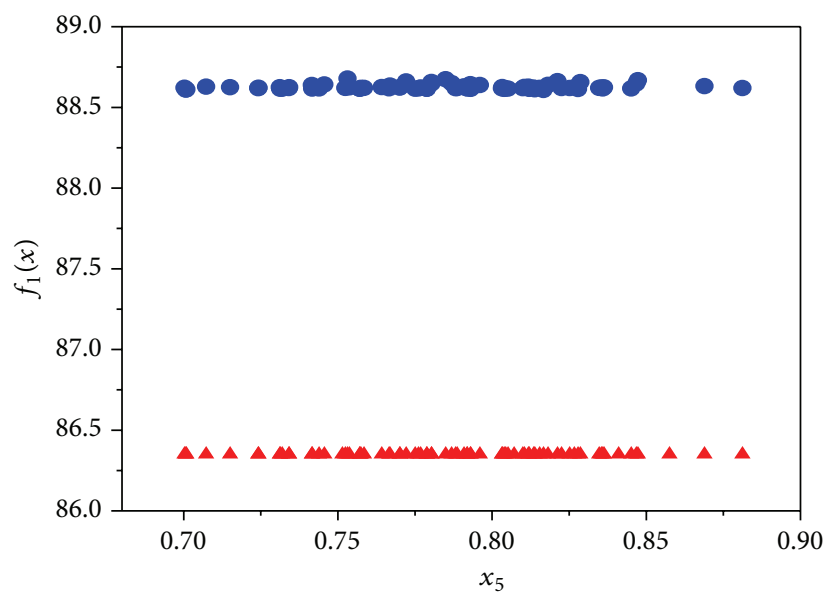

(a)

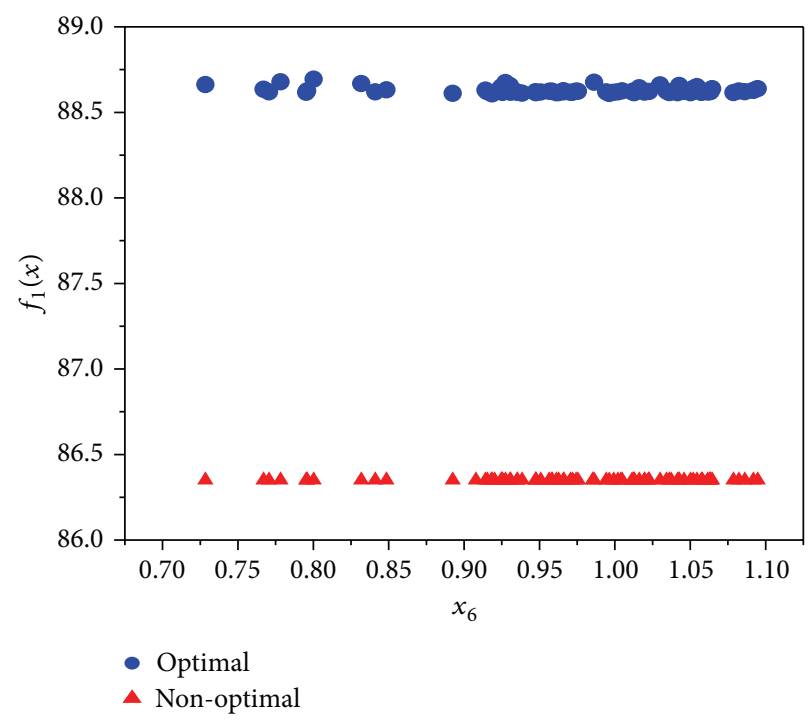

(c)

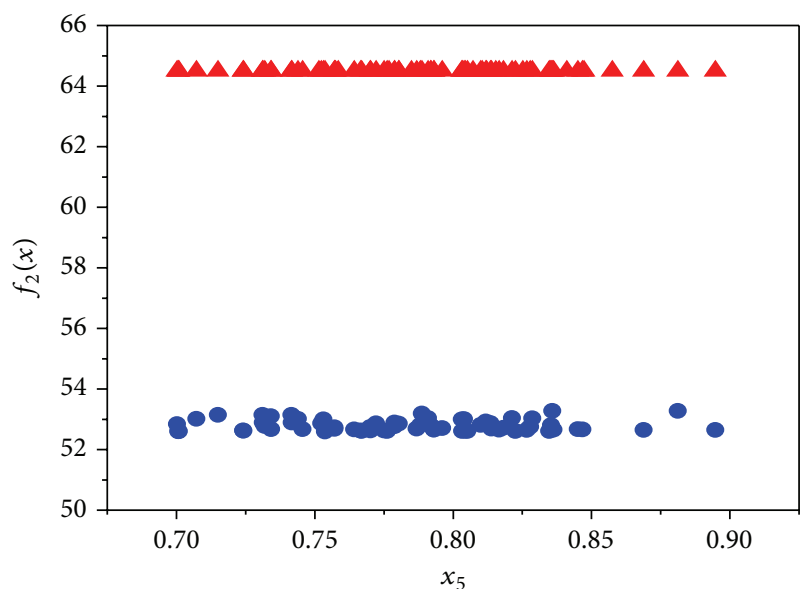

(b)

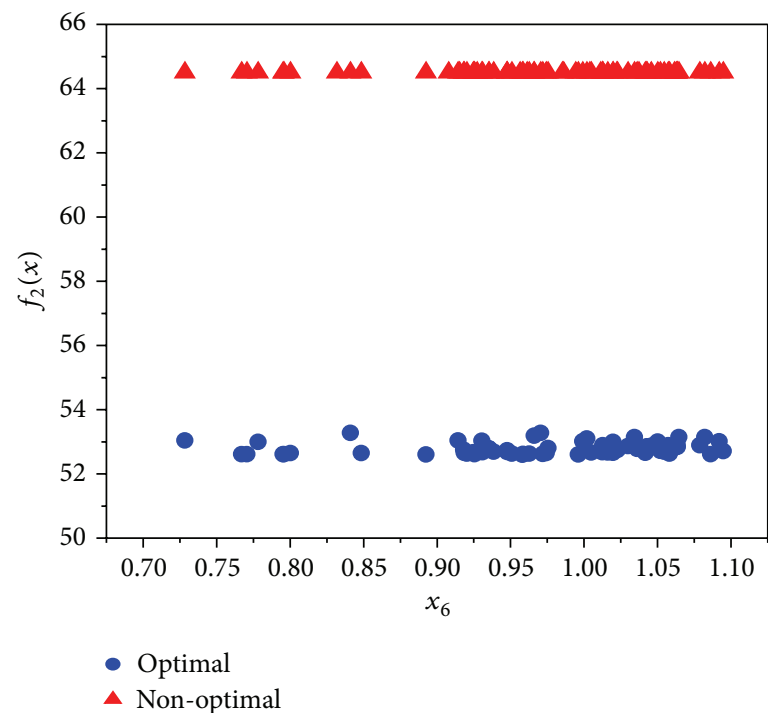

(d)

FIGURE 11: Pareto optimal set with regard to decision variables $x_{5}$ and $x_{6}$ with objectives $f_{1}(x)$ and $f_{2}(x)$.

nonoptimal operating conditions give $86.35 \%$ hydrocarbon recovery and $64.36 \mathrm{Gcal} / \mathrm{hr}$ of energy which shows a non proper region of operation. Applying this optimization model will approximately increase hydrocarbon recovery by $2.5 \%$ ( 235 tonnes of hydrocarbon/day) and minimize heat energy up to $16 \%$ of the non-optimal region.

To obtain optimal operating conditions for a desired percentage recovery and the corresponding minimal energy from Figure 9, the decision variables of the respective optimal set can be read off from Figures 10 and 11. Another set of Pareto front is generated when each objective is plotted against all the decision variables at the optimal solutions. This study provides an opportunity to process operators to choose the operating conditions needed to optimize both objectives.

Figures 10-11 show also a distinction between the optimal and non-optimal operating process variables of ADGAS plant, and its consistency is illustrated on all process variables. In these figures, the minimization and maximization objectives give a Pareto set below and above the non-optimal sets, respectively. Maximization of hydrocarbon recovery and minimization of energy are justified in the plots $f_{1}(x)$ and $f_{2}(x)$ versus $x_{1}, \ldots, x_{6}$ with the Pareto sets above and below the non-optimal set respectively. The difference in the optimal and non-optimal Pareto gives the amount of savings made on implementing this optimization approach. The Pareto set on the carbonate circulation rate $\left(x_{1}\right)$ takes on values close to the upper limit of its bounds. The carbonate reboiler duty $\left(x_{2}\right)$ also takes on optimal values near the upper bound of the constraint. Unlike decision variable $x_{1}$, the optimal values of $x_{2}$ vary significantly between its midpoint and the upper limit. The optimal value of decision variable $x_{3}$ takes values existing midway of the upper and lower bounds. Decision variables $x_{4}, x_{5}$, and $x_{6}$ span the entire feasible region. For all cases involving either $f_{1}(x)$ or $f_{2}(x)$, all variables give rise to the same trend.

\section{Conclusion}

This work presented a study to optimize a natural gas sweetening process with the objectives of maximization 


\section{Start up}

(i) Choose priori weights $w_{i}$

(ii) Combine objective functions using weighted sum method

(iii) Randomly generate an initial population: NP

(iv) $k=0$

Iteration

$$
\text { For each particle, } i=1 \text { to NP }
$$

For $d=1$ to $D$

$\mu_{i}^{d, k}=\frac{\left(\text { Pbest }_{i}^{d, k}+\text { Pgbest }_{i}^{d, k}\right)}{2}$

$\sigma_{i}^{d, k}=\mid P$ best $_{i}^{d, k}-$ Pgbest $_{i}^{d, k} \mid$

If $U[0,1]<0.5$ Then

$x_{i}^{d, k+1}=N\left(\mu_{i}^{d, k}, \sigma_{i}^{d, k}\right)$

Else

$x_{i}^{d, k+1}=$ Pbest $_{i}^{d, k}$

End if

End for

For each dimensiond $=1$ to $D$

IF $x_{i}^{d, k+1}<x_{\min }^{d}$ OR $x_{i}^{d, k+1}<x_{\max }^{d}$ Then

$x_{i}^{d, k+1}=x_{\min }^{d}+U[0,1] *\left(x_{\max }^{d}-x_{\min }^{d}\right)$

End if

End for

if $f\left(x_{i}^{d, k}\right)<f\left(\right.$ Pbest $\left._{i}^{d, k}\right)$ Then

Pgbest ${ }_{i}^{k}=x_{i}^{k+1}$

End if

$K=k+1$

Until termination criteria is satisfied

End for

Print Pgbest, $f_{i}$ (Pgbest)

Choose new weights $w_{i}$

Print a Pareto-front

Algorithm 1: The pseudocode of BBExp-PSO algorithm.

hydrocarbon recovery and minimization of heat energy required to run the process. ProMax simulation tool was used to model the process and the simulation results matched well the plant data. The multiobjective optimization using multiobjective bares-bone particle swarm indicates that a trade-off exists between the two objectives and plant operation can be optimized up to a $2.5 \%$ ( 235 tonnes/day) increase in hydrocarbon recovery and $16 \%$ decrease in heat required for the processoperation. The results also showed that the integration of an evolutionary algorithm in a process simulator can serve as an optimization tool box and can significantly improve the operation of the process. Future work will consider metamodeling of natural gas plants to overcome the large computational time involved in running integrated models of process simulators with evolutionary algorithms.

\section{Acknowledgments}

The authors would like to acknowledge GASCO, ADGAS, and Al Hosn Gas operating companies of Abu Dhabi National Oil Company (ADNOC) for their technical and financial support.

\section{References}

[1] S. A. Ebenezer and J. S. Gudmundsson, Removal of carbondioxide from natural gas for LNG production [Ph.D. thesis], Norwegian University of Science and Technology, Trondheim, Norway, 2005.

[2] A. Kohl and R. Nielsen, Gas Purification, Gulf, Houston, Tex, USA, 1997.

[3] R. Hubbard, "The role of gas processing in the natural-gas value chain," Journal of Petroleum Technology, vol. 61, no. 8, Article ID 118535, pp. 65-71, 2009.

[4] B. Hassan and T. Shamim, "Parametric and exergetic analysis of a power plant with $\mathrm{CO}_{2}$ and capture using chemical looping combustion," in Proceedings of the International Conference on Clean and Green Energy, Singapore, 2012.

[5] R. Mohammad, L. Schneiders, and J. Neiderer, "Carbondioxide capture from power plants: part I : a parametric study of the technical performance based on monoethanolamine," International Journal of Greenhouse Gas Control, vol. 1, no. 1, pp. 37-46, 2007.

[6] D. Wolbert, X. Joulia, B. Koehret, and L. T. Biegler, "Flowsheet optimization and Ootimal sensitivity analysis using exact derivatives,' Internal Report PA, 15213, Engineering Design Research Center, Carnegie Mellon University, Pittsburgh, $\mathrm{Pa}$, USA, 1993. 
[7] A. Aroonwilas, A. Chakma, P. Tontiwachwuthikul, and A. Veawab, "Mathematical modelling of mass-transfer and hydrodynamics in $\mathrm{CO}_{2}$ absorbers packed with structured packings," Chemical Engineering Science, vol. 58, no. 17, pp. 4037-4053, 2003.

[8] K. Y. Park and T.-W. Kang, "Computer simulation of $\mathrm{H}_{2} \mathrm{~S}$ and $\mathrm{CO}_{2}$ absorption processesabsorption processes," Korean Journal of Chemical Engineering, vol. 12, no. 1, pp. 29-35, 1995.

[9] M. R. Rahimpour and A. Z. Kashkooli, "Enhanced carbon dioxide removal by promoted hot potassium carbonate in a split-flow absorber," Chemical Engineering and Processing, vol. 43, no. 7, pp. 857-865, 2004.

[10] M. R. Rahimpour and A. Z. Kashkooli, "Modeling and simulation of industrial carbon dioxide absorber using aminepromoted potash solution," Iranian Journal of Science and Technology B, vol. 28, no. 6, pp. 653-666, 2004.

[11] K. Y. Park and T. F. Edgar, "Simulation of the hot carbonate process for removal of $\mathrm{CO}_{2}$ and $\mathrm{H}_{2} \mathrm{~S}$ from medium Btu gas," Energy progress, vol. 4, no. 3, pp. 174-181, 1984.

[12] P. Patil, Z. Malik, and M. Jobson, "Retrofit design for gas sweetening processes," in Proceedings of the IChemE Symposium, 152, pp. 460-467, 2006.

[13] J. C. Polasek and J. A. Bullin, "Design and optimization of integrated amine sweetening, claus sulfur and tail gas cleanup units by computer simulation," Internal Report, Bryan Research \& Engineering, Bryan, Tex, USA, 1990.

[14] J. C. Polasek, J. A. Bullin, and S. T. Donnely, "Alternative flow schemes to reduce capital and operating costs of amine sweetening units," Internal Report, Bryan Research \& Engineering, Bryan, Tex, USA, 2006.

[15] R. H. Weiland, M. Rawal, and R. G. Rice, "Stripping of carbondioxide from monoethanolamine solutions in a packed column," AIChE Journal, vol. 28, no. 6, pp. 963-973, 1982.

[16] H. Pierreval and L. Tautou, "Using evolutionary algorithms and simulation for the optimization of manufacturing systems," IIE Transactions, vol. 29, no. 3, pp. 181-189, 1997.

[17] E. Zitzler and L. Thiele, "Multiobjective optimization using evolutionary algorithms: a comparatice case study," in Proceedings of the 5th International Conference on Parallel Problem Solving from Nature (PPSN '98), Amsterdam, The Netherlands, 1998.

[18] S. Dehuri and S.-B. Cho, "Multi-criterion Pareto based particle swarm optimized polynomial neural network for classification: a review and state-of-the-art," Computer Science Review, vol. 3, no. 1, pp. 19-40, 2009.

[19] A. Konak, D. Coit, W. Smith, and E. Alice, "Multi-objective optimization using genetic algorithm: a tutorial," Reliability Engineering \& System Safety, vol. 91, pp. 992-100, 2006.

[20] L. Mian, S. Azarm, and V. Aute, "A multi-objective genetic algorithm for robust design optimization," in Proceedings of the Genetic and Evolutionary Computation Conference (GECCO '05), pp. 771-778, New York, NY, USA, June 2005.

[21] W. Wu, Y. Liou, and Y. Zhou, "Multiobjective optimization of a hydrogen production system with low $\mathrm{CO}_{2}$ emissions," Industrial and Engineering Chemistry Research, vol. 51, no. 6, pp. 2644-2651, 2012.

[22] R. T. Marler and J. S. Arora, "The weighted sum method for multi-objective optimization: new insights," Structural and Multidisciplinary Optimization, vol. 41, no. 6, pp. 853-862, 2010.

[23] R. T. Marler and J. S. Arora, "Survey of multi-objective optimization methods for engineering," Structural and Multidisciplinary Optimization, vol. 26, no. 6, pp. 369-395, 2004.
[24] E. Bernier, F. Maréchal, and R. Samson, "Multi-objective design optimization of a natural gas-combined cycle with carbon dioxide capture in a life cycle perspective," Energy, vol. 35, no. 2, pp. 1121-1128, 2010.

[25] H. Li, F. Maréchal, M. Burer, and D. Favrat, "Multi-objective optimization of an advanced combined cycle power plant including $\mathrm{CO}_{2}$ separation options," Energy, vol. 31, no. 15, pp. 31173134, 2006.

[26] M. M. Montazer-Rahmati and R. Binaee, "Multi-objective optimization of an industrial hydrogen plant consisting of a $\mathrm{CO}_{2}$ absorber using DGA and a methanator," Computers and Chemical Engineering, vol. 34, no. 11, pp. 1813-1821, 2010.

[27] L. Sun and H. H. Lou, "A strategy for multi-objective optimization under uncertainty in chemical process design," Chinese Journal of Chemical Engineering, vol. 16, no. 1, pp. 39-42, 2008.

[28] O. O. Shadiya, V. Satish, and K. A. High, "Process enhancement through waste minimization and multiobjective optimization," Journal of Cleaner Production, vol. 31, pp. 137-149, 2012.

[29] K. E. Parsopoulos and M. N. Vrahatis, "Recent approaches to global optimization problems through particle swarm optimization," Natural Computing, vol. 1, pp. 230-235, 2002.

[30] R. Poli, J. Kennedy, and T. Blackwell, "Particle swarm optimization," Swarm Intelligence, vol. 1, pp. 33-35, 2007.

[31] M. Reyes-Sierra and C. A. Coello, "Multi-objective particel swarm optimizers: a survey of the state-of-the-art," International Jounal of Computing Intelligenece Research, vol. 2, pp. 287300, 2006.

[32] J. Kennedy and R. Eberhart, "Particle swarm optimization," in Proceedings of the IEEE International Conefercence on Neural Networks, vol. 4, pp. 1942-1948, December 1995.

[33] H. Zhang, D. Kennedy, G. Rangaiah, and A. Bonilla-Petriciolet, "Novel bare-bones particle swarm optimization and its performance for modeling vapor-liquid equilibrium data," Fluid Phase Equilibria, vol. 301, no. 1, pp. 33-45, 2011.

[34] H. E. Benson and R. W. Parrish, "HiPure process removes $\mathrm{CO}_{2} / \mathrm{H}_{2} \mathrm{~S}$," Hydrocarbon Processing, vol. 53 , no. 4 , pp. 81-82, 1974.

[35] Bryan Research and Engineering, "ProMax V3.2," 2010, http:// www.bre.com/home.aspx.

[36] J. Kennedy, "Bare bones particle swarms," in Proceedings of the IEEE Swarm Intelligence Symposium, pp. 80-87, 2003. 


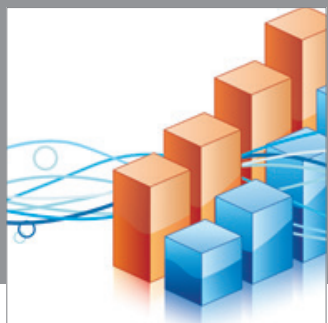

Advances in

Operations Research

mansans

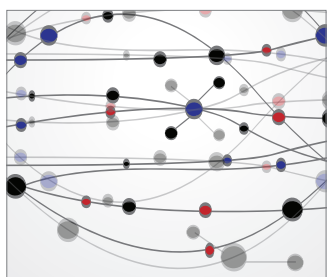

The Scientific World Journal
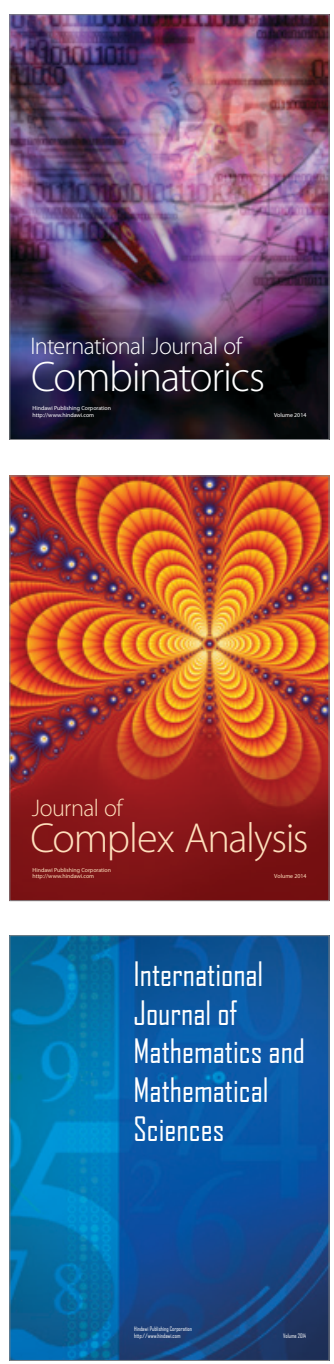
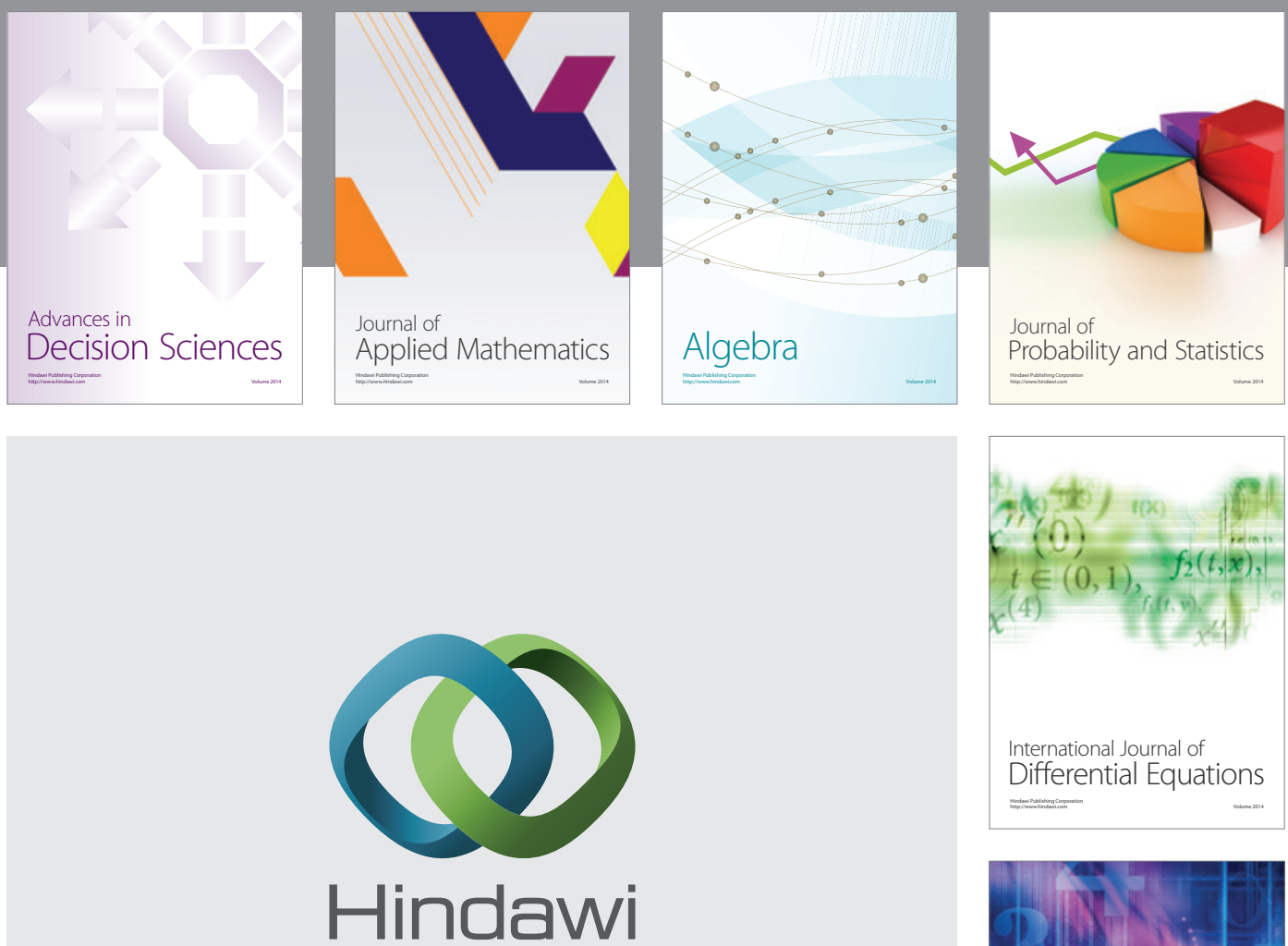

Submit your manuscripts at http://www.hindawi.com
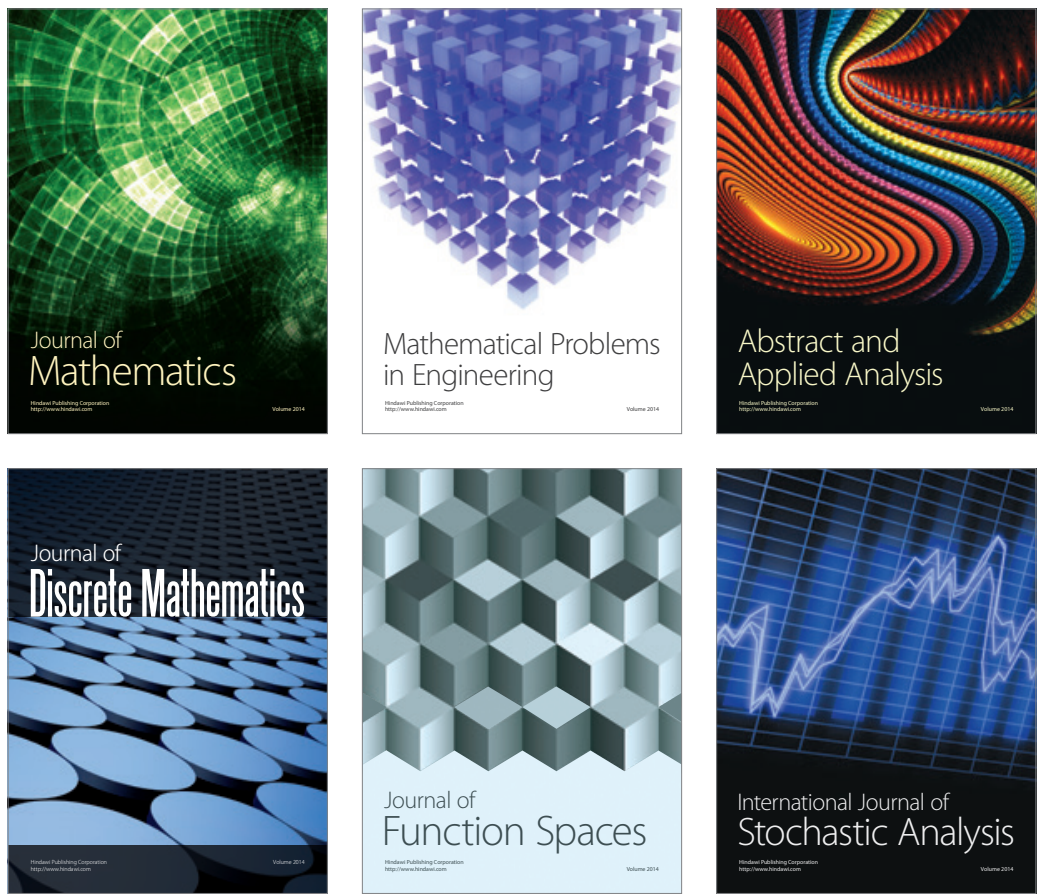

Journal of

Function Spaces

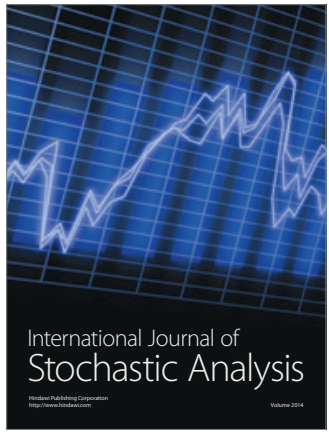

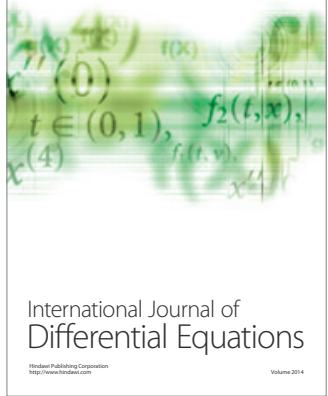
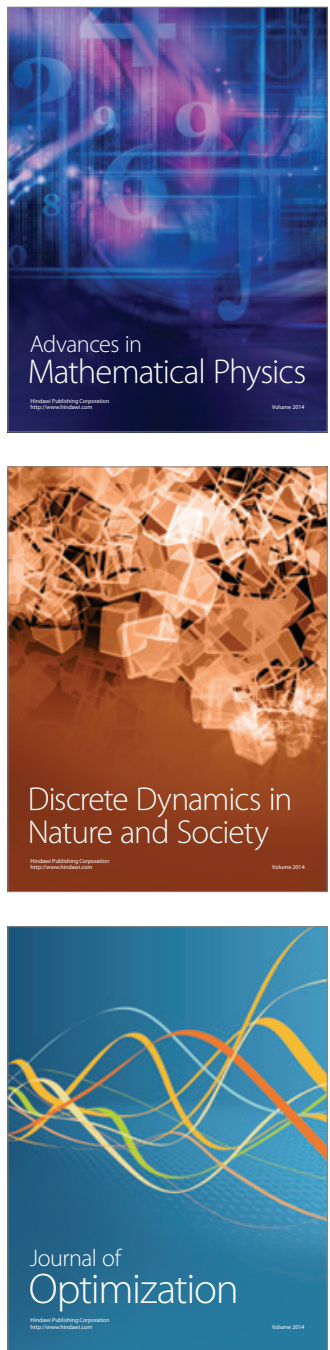